\title{
Intelligent Boiler Fault Monitoring Interface and Advisory Guide
}

\author{
Nong Nurnie Mohd Nistah ${ }^{+}$, Lim King Hann, Lenin Gopal \\ Curtin University Malaysia \\ Miri Sarawak, Malaysia
}

\begin{abstract}
Monitoring boiler faulty condition in a huge power generating plant can be a challenging task in the operation. The structural complexity of the boiler unit makes it difficult for plant operators to quickly analyze any abnormality in its sensor reading. In this paper, an intelligent interface using artificial neural network is proposed to locate faults by providing instant advisory guide. The interface is developed to generate an advisory guide for plant operators as the point of reference to carry out maintenance task once a fault is identified. With reference to the simulation result, possible faults were recorded to have occurred at a specific time interval. The proposed interface was able to identify abnormality of operational parameters at the confident level of $\pm 6.3 \%$. This information is important as a guideline for an effective inspection and maintenance work.
\end{abstract}

Keywords: Graphical user interface; Advisory guide, Intelligent monitoring

\section{Introduction}

An Intelligent Monitoring Interface (IMI) is an extension of a human-machine interface system that tracks and improves the responses of an event associated with monitored equipment [1], [2]. It enables user to perform potentially complex tasks more effectively and quickly with greater accuracy. This is made possible by presenting users with information on the equipment condition, user's next actions, and warnings of undesirable consequences and suggestions of an alternative action. A power plant boiler monitoring system does not allow simultaneous collaboration and direct interaction between plant operators and the system offsite.

An extension to the current monitoring system is the Graphical User Interface (GUI) development for both client side data representation and control and maintenance operations. The most common interface implementation of a monitoring system is through an on-site server where authenticated user access the data collected through their local workstation connected to a Virtual Private Network (VPN). Meanwhile, a remote server monitoring system allows data collected on site to be sent to a remote server and accessible through any standard web browsers (e.g. Mozilla, Explorer, or Google Chrome). Anyone with an authenticated username and password can view the data without installing the client software. The advantage of the latter; plant staff, analyst and equipment and maintenance operators and technicians can simultaneously look at the data to collaborate on remedial actions [3]. Rather than delivering machine data in-house only (on-site control rooms), the Internet allows information to be accessible from anywhere, anytime.

\section{Boiler Fault Monitoring Interface Design}

The steps involved in designing the GUI of the advisory guide of the monitoring system is elaborated in detailed in this section. The preliminary design of the GUI is based on Figure 1. Ideally, plant operators of this monitoring system are made accessible at different levels, and possibly different types of views and control, depending on their profile, conditions of the system and the current task being executed.

\footnotetext{
Corresponding author. Tel.: +6085443939 ext.: 2448

E-mail address: nong.nurnie@curtin.edu.my
} 


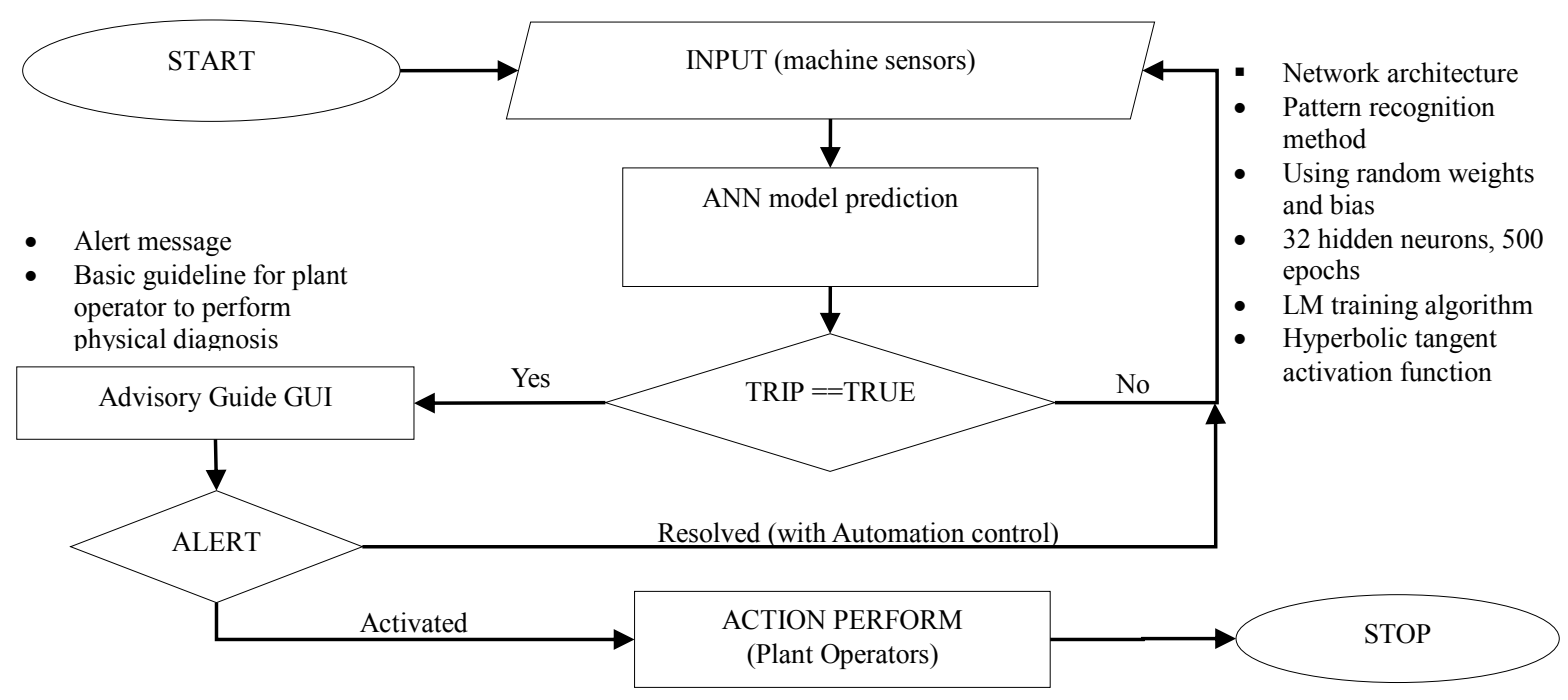

Fig. 1: Flow chart of the monitoring system.

A user interface is used to represent the functionality of the system by categorizing user actions and their working paradigms into interactive visual objects on a user's screen or monitor. These important features of a user interface make a system easier to use [4], [5]. Visualization and interactivity are important aspects when designing a monitoring system interface. This is because the graphical capabilities of a computer using images, buttons or animation, helps the user to easily navigate the system. Furthermore, the intractability between the system and the user allows simultaneous response to any changes or feedback of the system. This rich visual content allows user to gain useful practical insights into the monitoring systems [6]. To define the best fitting user interface for the linked power plant equipment sensors and trip mechanism, a set of basic requirements needs to be considered. They include the following; the knowledge specification of the monitored system, an executable interface must be logically possible for the specified requirements, it has to be feasible to verify properties and validate the given specifications, it must be possible to classify users of the interface, it must be possible to define the tasks that will be made available only to a specific user profile.

As for the human factor, the main users' profile that needs to be defined are Expert, Maintenance, Supervisor and Operators. An expert will be the direct person to contact for an alarm event involving an equipment sensor malfunctions or trips. The purpose of the user interface is to provide the expert on the equipment sensor installation, Artificial Neural Network (ANN) design, and testing and diagnosis report. Maintenance on the other hand refers to the user interface that will be used to display statistics or machine reading for various parameter measurements for the maintenance team. The task of a supervisor is to manage the machinery processes and oversee the learning process of the embedded ANN into the system. Hence, the user interface will provide information regarding the movements of the plant equipment operators and the communication link and channel for information exchange between the two personnel. While operators are generally the one handling the continuous innovation and improvements of the machinery. Furthermore, should there be any need for equipment changeover and reporting anomalies during inspections; direct communication to the supervisors are critical especially for triggered alarm or trip inspections. The user interface should provide the interaction medium in real time. These requirements are known as the Human Machine Interaction (HMI). It provides the link between the sensory capturing system to the end user by providing the means for a long term analysis and aid improvements and innovation of the equipment [7], [8].

\section{Design of Advisory Guide}

In 2002, an expert system to manage abnormal condition of a manufacturing system has already been proposed by [9] where their main objective was to develop a system that was able to detect unusual events early, assess the potential impacts of the identified event, diagnose the root cause and provide operators with guided advice for an appropriate immediate actions.

Noureldin and Roveta [9] applied "smart" generic and reusable objects known as "Generic Heaters" developed using Gensym Optegrity software to provide proactive diagnostic to manage identified abnormality and performance indices. Generic Heaters are software objects containing diagnostic, fault models and advisory messages for managing over 80 faults typical for boilers, furnaces, ethylene crackers and incinerators. For this paper however, to use their method for a 32-sensor parameter reading to identify 
the fault condition of a boiler will require over 2,560 fault models objects to consider. This will be time consuming and may not be practical for real time implementation.

\subsection{The plant boiler parameter fault identifier}

The plant boiler parameter fault identifier was developed using a multi layered perceptron ANN model as shown in Figure 2. As an experimental setup, 10 datasets was used to train the network with 500 epochs and random weights using the Levenberg-Marquardt training algorithm and hyperbolic tangent (Tanh) activation function. The model was able to classify fault reading from 32 parameter input data fairly well, with an average of less than 10\% misclassified error (MCR) [10]. The fault reading are then sent to the GUI for plant operators to be viewed, analyze and recorded. The input parameters used for this simulation are labeled according to the corresponding temperature pressure and flow rate of the boiler under study in Table 1.

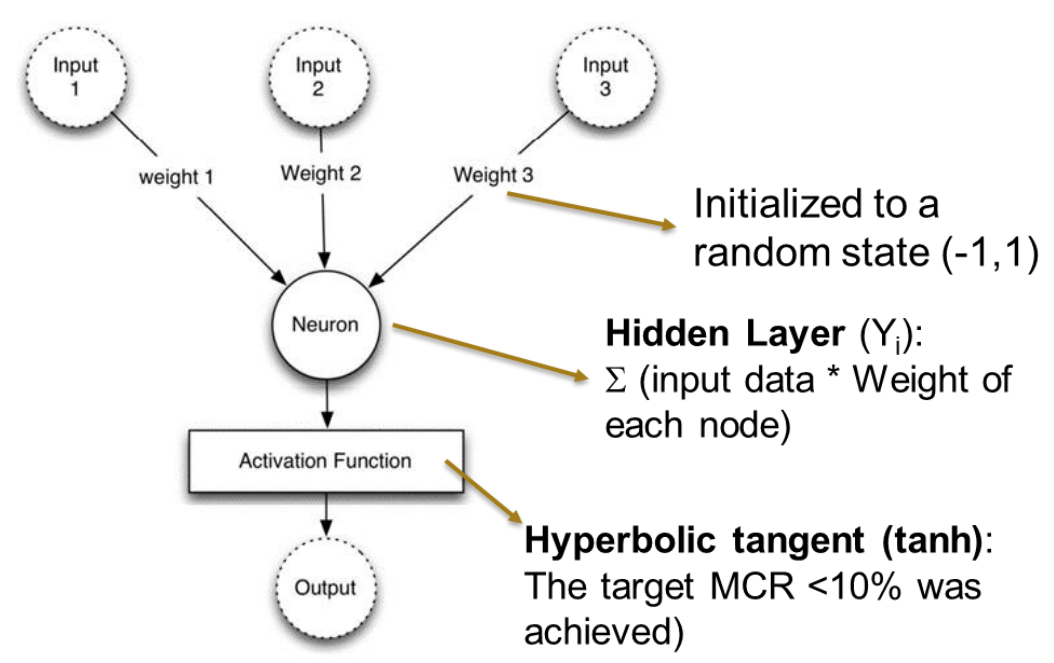

Fig. 2: Multi-layered perceptron ANN model for the intelligent monitoring interface

Table 1: Boiler Operational Parameter and Data Description

\begin{tabular}{|c|c|c|}
\hline Parameter List & Description & Unit \\
\hline V1 & Total combined steam flow & ton/hr \\
\hline $\mathbf{V 2}$ & Feed water flow & ton/hr \\
\hline V3 & Boiler drum pressure & bar \\
\hline V4 & Super heater steam pressure & bar \\
\hline $\mathbf{V 5}$ & Super heater steam temperature & ${ }^{\circ} \mathbf{C}$ \\
\hline V6 & High temperature re-heater outlet temperature & ${ }^{\circ} \mathbf{C}$ \\
\hline $\mathbf{V 7}$ & High temperature super heater exchange metal temperature & ${ }^{\circ} \mathbf{C}$ \\
\hline V8 & Intermediate temperature (A) super heater exchange metal temperature & ${ }^{\circ} \mathbf{C}$ \\
\hline V9 & High temperature super heater inlet header metal temperature & ${ }^{\circ} \mathrm{C}$ \\
\hline V10 & Final super heater outlet temperature & ${ }^{\circ} \mathbf{C}$ \\
\hline V11 & Super heater steam pressure transmitter (control) & bar \\
\hline V12 & Feed water valve station & ton/hr \\
\hline V13 & Feed water control valve position & $\%$ \\
\hline V14 & Drum level corrected (control) & $\mathbf{m m}$ \\
\hline V15 & Drum level compensated (from protection) & $\mathbf{m m}$ \\
\hline V16 & Feed water flow transmitter & $\%$ \\
\hline V17 & Boiler circulation pump 1 pressure & bar \\
\hline V18 & Boiler circulation pump 2 pressure & bar \\
\hline V19 & Low temperature super heater left wall outlet before super heater dryer & ${ }^{\circ} \mathbf{C}$ \\
\hline V20 & Low temperature super heater right wall outlet before super heater dryer & ${ }^{\circ} \mathrm{C}$ \\
\hline V21 & Low temperature super heater left wall outlet after super heater dryer & ${ }^{\circ} \mathrm{C}$ \\
\hline V22 & Low temperature super heater right wall exchange metal temperature & ${ }^{\circ} \mathrm{C}$ \\
\hline V23 & Intermediate temperature (B) super heater exchange metal temperature & ${ }^{\circ} \mathbf{C}$ \\
\hline V24 & Intermediate temperature super heater outlet before super heater dryer & ${ }^{\circ} \mathrm{C}$ \\
\hline V25 & Intermediate temperature super heater outlet header metal temperature & ${ }^{\circ} \mathbf{C}$ \\
\hline V26 & High temperature super heater outlet header metal temperature & ${ }^{\circ} \mathrm{C}$ \\
\hline V27 & High temperature re-heater outlet steam pressure & bar \\
\hline V28 & Superheated steam form intermediate temperatures outlet pressure & bar \\
\hline V29 & Super heater water injection compensated flow & ton/hr \\
\hline V30 & Economizer inlet pressure & bar \\
\hline V31 & Economizer inlet temperature & ${ }^{\circ} \mathrm{C}$ \\
\hline V32 & Economizer outlet temperature & ${ }^{\circ} \mathrm{C}$ \\
\hline
\end{tabular}




\subsection{The plant boiler fault advisory guide}

The advisory guide is generated to provide detailed explanation of the root cause of the fault. It will also provide a list of suggestions of maintenance actions to be carried out to address the faults. As shown in Figure 3, an output of a normalized data reading for the observed 32 parameters with a 1-minute intervals. The GUI also include a section with a warning and an alarm indicator that counts the number of abnormal/fault readings identified. Other features added in the GUI, is an update button, where for each button press; a new set of time stamp data is retrieved from the fault identifier and displayed in the first column for viewing. Another button to generate the text file of the advisory guide is also included. The action performed when the button is pressed is it will open a text file that documents the fault reading. The description of the related parameters of the fault reading will be listed along with suggestion of maintenance task.

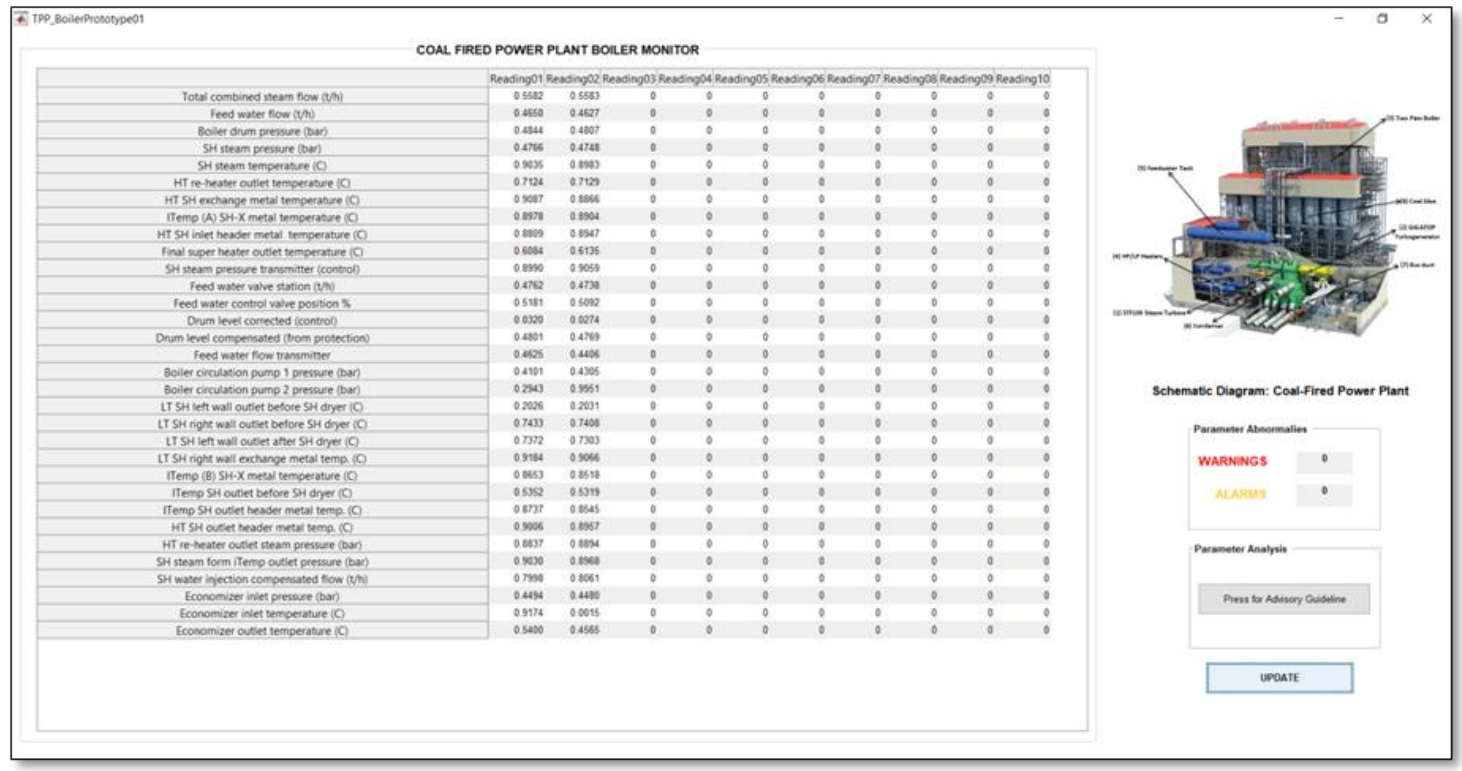

Fig. 3: A sample of the GUI with a time stamp parameter reading for the boiler advisory guide.

\section{Boiler Parameter Sensitivity Analysis}
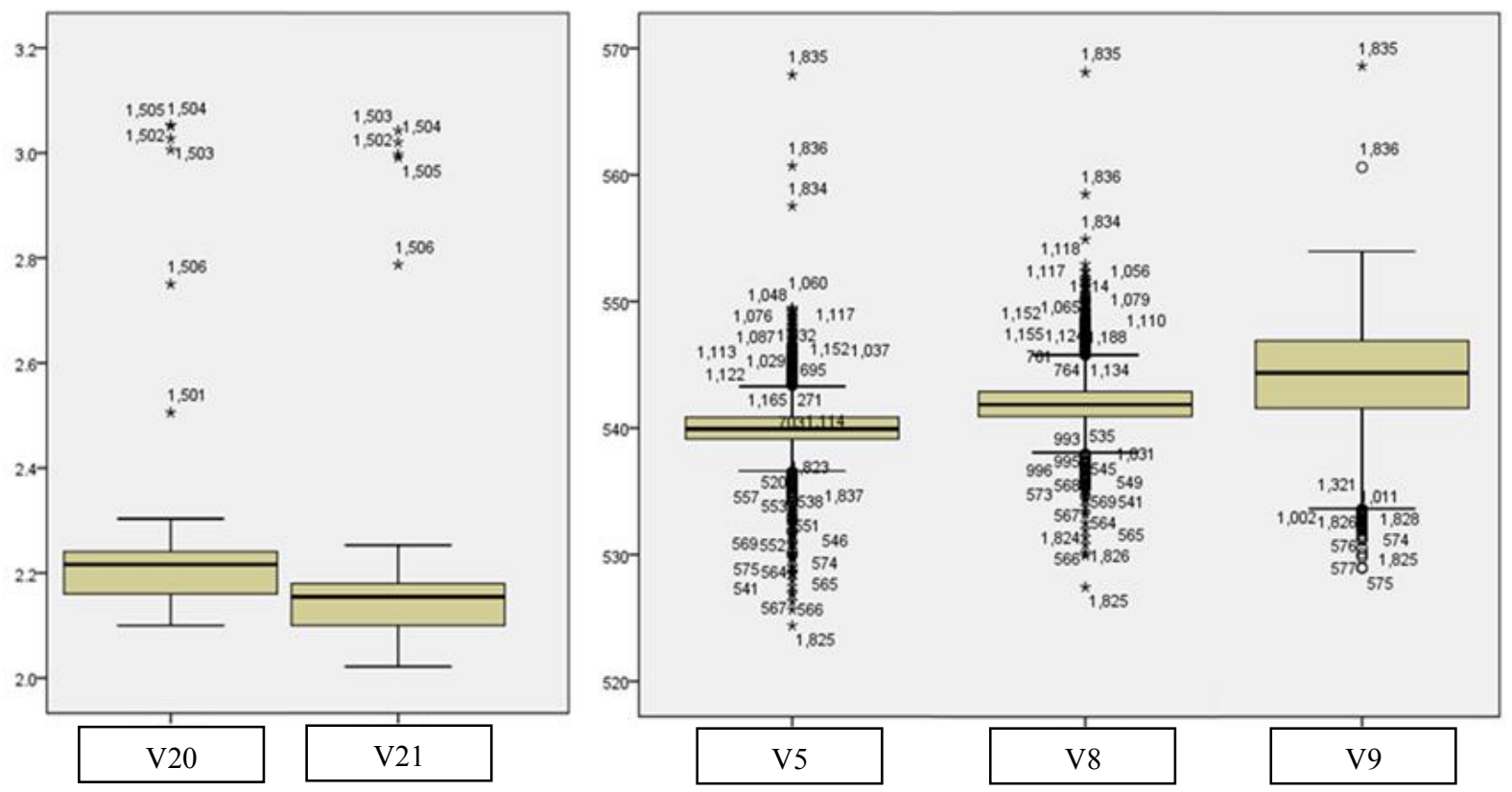

Fig. 4: A sample of the outliers identified in the sensitivity analysis for five significant parameters in one of the fault data reading.

To validate the effect of each of the 32 parameters monitored in the system on the boiler performance, a sensitivity analysis was carried out. The outcome of one of the fault analysis is illustrated in Figure 4. As illustrated here in the boxplots, there are a group of outliers identified between the 1501 to 1508 minute 
intervals for V20 (low temperature superheater right wall outlet before superheater dryer) and V21 (low temperature superheater left wall outlet after superheater dryer). This could be an indicator that the low super heater wall outlet parameters may be experiencing some disturbances causing some faulty reading which is about $\pm 6.3 \%$ exceeding the lower and upper bounds of the confidence intervals.

Apart from these two parameters, three other parameters related to the super heater temperature were also showing a slight increment in its reading at intervals 1835 to 1837, as demonstrated in Figure 5. They are V5, which is the super heater steam temperature, V8 the intermediate temperature (A) super heater exchange metal temperature, and V9 as the high temperature super heater inlet header metal temperature which were above the upper bounds of the confidence interval of $544.8^{\circ} \mathrm{C}, 567.7^{\circ} \mathrm{C}$, and $570.2^{\circ} \mathrm{C}$ respectively. The correlation between these variables are unclear when analysed with a statistical tool, hence the importance to use neural network to make the correlation between these variable reading.

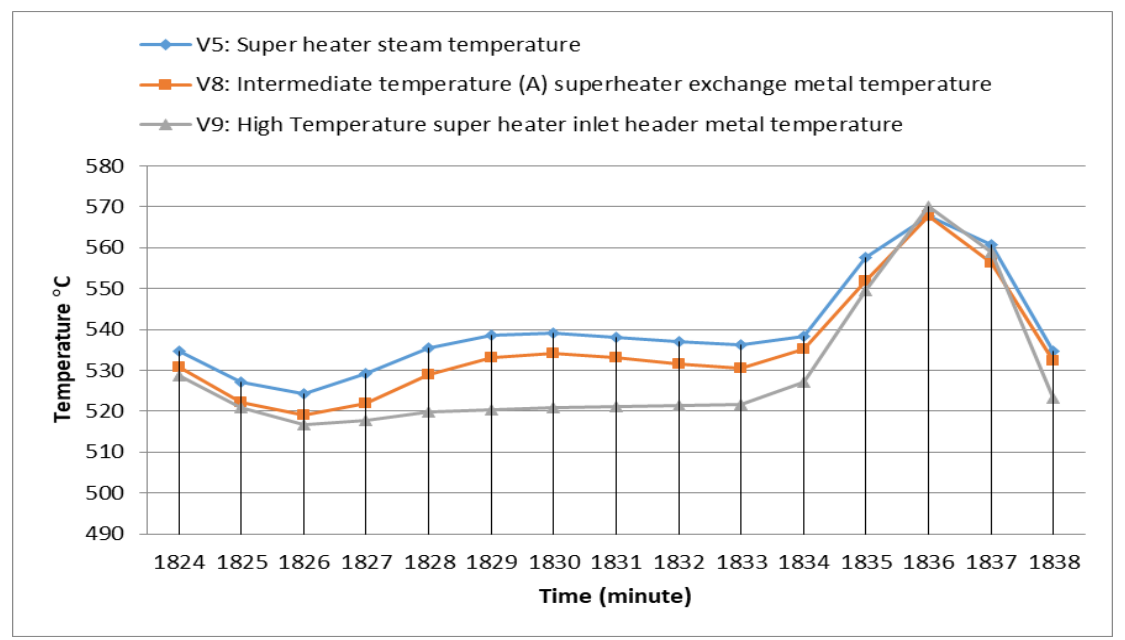

Fig. 5: V5, V8 and V9 with a sudden upsurge of temperature reading at interval 1835 to 1837.

\section{Conclusion}

Intelligent advisory guide is proposed to be added to the existing monitoring system not only benefit the industry economically, but it may also improve the overall optimization of the boiler equipment unit in a power plant. The advisory guide alerts an abnormal condition in the boiler using a multilayer perceptron prediction tool that predicts and help to avoid abnormalities. It can save thousands in revenue as well as the lives of people involving in the work of maintaining the normal boiler operation of the power plant. In the future, intermediate parameters such as the increased and decreased rate of change will be inserted for boiler plant monitoring.

\section{References}

[1] B. Hayes-roth, R. Washington, and W. Road, "Intelligent Monitoring and Control," pp. 243-249.

[2] A. Agah and K. Tanie, "Intelligent graphical user interface design utilizing multiple fuzzy agents," Interact. Comput., vol. 12 , no. 5, pp. 529-542, 2000.

[3] N. Baxter, "Remote Machine Monitoring: A Developing Industry," no. June 2006, 2008.

[4] A. Isabella and J. E. Retna, "Study Paper on Test Case Generation for Gui Based Testing," Int. J. Softw. Eng. Appl., vol. 3, no. 1, pp. 139-147, 2012.

[5] Z. Singhera, E. Horowitz, and A. Shah, “A Graphical User Interface (GUI) Testing Methodology,” Int. J. Inf. Technol. Web Eng., vol. 3, no. 2, pp. 1-18, 2008.

[6] J. Chacón, G. Farias, H. Vargas, A. Visioli, and S. Dormido, "Remote Interoperability Protocol: A bridge between interactive interfaces and engineering systems," IFAC-PapersOnLine, vol. 48, no. 29, pp. 247-252, 2015.

[7] M. Blanton et al., "Human-Computer Interaction," Encycl. Database Syst., pp. 1327-1331, 2009.

[8] K. Martinsen, J. Downey, and I. Baturynska, "Human-Machine Interface for Artificial Neural Network Based Machine Tool Process Monitoring,” Procedia CIRP, vol. 41, pp. 933-938, 2016.

[9] H. Noureldin and F. Roveta, "Using Expert System and Object Technology for Abnormal Condition Management," 2002.

[10] N. N. Mohd Nistah, K. H. Lim, L. Gopal, and F. B. I. Alnaimi, "Coal-Fired Boiler Fault Prediction Using Artificial Neural Networks," Int. J. Electr. Comput. Eng., vol. 8, no. 1, 2018. 